\title{
Suspecting the unsuspected in a large adnexal mass in a postmenopausal woman
}

\author{
Manisha Jain $^{1}{ }^{*}$, Jyoti Nath Modi ${ }^{1}$, Uma Chourasia ${ }^{1}$, Ritu Singhal ${ }^{2}$ \\ ${ }^{1}$ Department of Obstetrics \& Gynaecology, People's College of Medical Sciences \& Research Centre, People's \\ University, Bhopal, Madhya Pradesh, India \\ ${ }^{2}$ Department of Pathology, Jawahar Lal Nehru Cancer Hospital, Bhopal, Madhya Pradesh, India
}

Received: 24 September 2013

Accepted: 8 October 2013

*Correspondence:

Dr. Manisha Jain,

E-mail: mnishu2002@yahoo.com

(C) 2013 Jain M et al. This is an open-access article distributed under the terms of the Creative Commons Attribution Non-Commercial License, which permits unrestricted non-commercial use, distribution, and reproduction in any medium, provided the original work is properly cited.

\begin{abstract}
Adnexal mass in postmenopausal woman is a diagnostic challenge. Due to the age, concern for malignancy is always there. At times however, despite the usual investigations, diagnosis remains elusive till histopathology report is at hand. To add to the enigma, ovarian masses in postmenopausal woman may remain silent till quite large and even among those which are symptomatic a predictable presentation may not be there. A careful clinical examination with a high degree of suspicion of clinical variants is thus recommended while dealing with ovarian tumors in postmenopausal women.
\end{abstract}

Keywords: Postmenopausal, Adnexal mass, Sertoli cell tumor

\section{INTRODUCTION}

Adnexal masses in postmenopausal age group, often detected incidentally, are usually a clinical dilemma. The diagnostic evaluation of such masses primarily aims at exclusion of malignancy. However, despite a thorough work- up the diagnosis may remain elusive until a histopathology report is available. The commonest lesions in such clinical presentations are ovarian neoplasms, with epithelial tumors being the predominant variety. The nonepithelial ovarian tumors, mainly germ cell and stromal cell tumors account for about $10 \%$ of all ovarian neoplasms. ${ }^{1}$

Among the non-epithelial ovarian tumors, the Sex cord stromal tumor are a rare variety derived from ovarian stromal structures and sex cord of embryonic gonad giving rise to more specialized cells as sertoli, leydig or thecagranulosa cells. Typically, they are unilateral, confined to the ovary and are predominantly seen in $2^{\text {nd }}$ and $3^{\text {rd }}$ decade of life. ${ }^{1}$ These uncommon tumors account for about 3-5\% of ovarian neoplasms and $7 \%$ of ovarian malignancies. ${ }^{2}$ Many are functional and hormones thus released may cause virilization or symptoms related to estrogen excess giving an early clue. However, morphologic variants are common which can generate difficulty in diagnosis until operated upon.

\section{CASE REPORT}

A 61 year old lady, postmenopausal since last eight years, presented to Gynaecology OPD with vague abdominal discomfort and undocumented low grade fever since the preceding two months. She also complained of constipation. There were no urinary symptoms. Her menstrual cycles in the past were normal. She had had three term vaginal deliveries in the past and the last childbirth was thirty-two years ago. She was detected with hypertension two years ago and was on medications since then.

On examination, she was of average built, ambulatory and in good general condition. Her vital parameters were within normal limits with the blood pressure being 140/80. She had mild pallor, no icterus, oedema or lymphadenopathy. Her cardiovascular and respiratory system examination were within normal limits. On perabdominal examination, there was a large firm, non-tender mass arising out of pelvis (almost the size of a 20 week 
size gravid uterus), with restricted side-to-side mobility. There was no ascites and the skin over the mass was normal and non adherent. Rest of the abdomen including all hernial orifices appeared normal. On per speculum examination, cervix was high and appeared pulled up, grossly normal looking. On per-vaginum examination, the same mass was felt through post and right fornix, in continuity with the uterus, no tenderness. On per-rectal examination, the rectal mucosa was free and there was no mass or deposit palpable in the pouch of Douglas.

A provisional admitting diagnosis of fibroid uterus was made with a differential diagnosis of malignant ovarian tumor.

The routine baseline blood and urine investigations were within normal limits. The sonography suggested a large malignant ovarian growth closely adherent to right lateral and posterior wall of the uterus. Uterus showed heterogeneous endomyometrial echo pattern with small amount of collection in the cervical canal. Bilateral ovaries could not be made out. A CT scan whole abdomen was done which reported the large well defined soft tissue mass lesion in the pelvic cavity with heterogenous enhancement and multiple pockets of necrotic areas within it while uterus appeared to be elongated with significant collection in the endocervical region. CT suggested of neoplastic pelvic mass possibly ovarian. The serum CA125 was 29.20 $\mathrm{IU} / \mathrm{ml}$ and the CEA was $0.63 \mathrm{ng} / \mathrm{ml}$. The pap smear was negative for malignancy or dysplasia. The Fine Needle Aspiration Cytology (FNAC) suggested benign spindle cell lesion with scant cellularity and lipoid background.

An exploratory laparotomy was planned. Intra operative peritoneal washings were taken which were negative for malignancy. The omentum was adherent to parieties. Uterus was normal sized and elevated almost to the level of pelvic brim by the mass arising from the right cornu. This mass was a hard, fixed vascular mass about $20 \times 15 \mathrm{~cm}$ in size and it extended laterally as well as downward and into the retroperitonium. It invaded into the rectovaginal space and into the retroperitoneal space beneath the leaves of broad ligament almost engulfing the right uterine artery and the right ureter. There were areas of necrosis and degeneration within the mass. It was difficult to make out the tubes and ovary separately on both sides. There were no peritoneal surface deposits or enlarged lymph nodes. The capsule of the mass was densely adhered to the surrounding tissue and bowel so an intracapsular piecemeal resection of the mass with subtotal hysterectomy could only be done. General Surgeons were also called for expert help. The specimen thus removed was sent for histopathological and immunohistochemical studies. An Intraperitoneal drain was placed.

Her post operative period was unremarkable except for a slightly prolonged period of postoperative ileus which was managed conservatively. She subsequently recovered well and was discharged on $14^{\text {th }}$ postoperative period in good condition.
The histopathology reported presence of partially encapsulated solid tumor comprising of moderately differentiated tubules lined by cuboidal and spindle cells with ovoid nuclei and bland chromatin. The tubules were separated by dense fibrous stroma. There were no leydig cells. The endometrial lining of the uterus showed simple hyperplasia without atypia. Immunohistochemistry studies were undertaken which showed focal positivity for EMA and pancytokeratin. The tumor was weakly positive for Vimentin while negative for Calretinin stain. The final diagnosis of moderately differentiated sertoli cell tumor was made. Patient was advised chemotherapy (BEP) as further management.

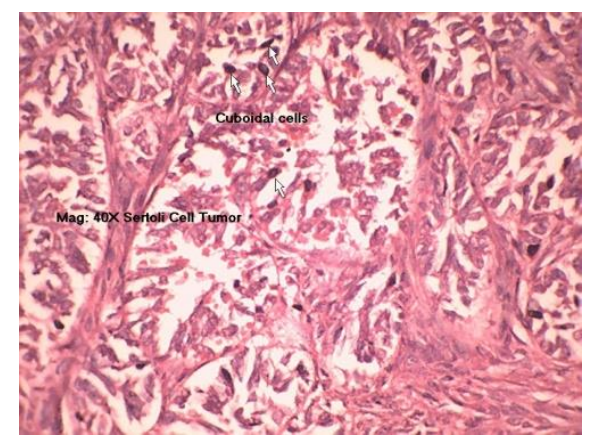

Figure 1: Tubules lined by cuboidal and spindle cell with ovoid nuclei and bland chromatin.

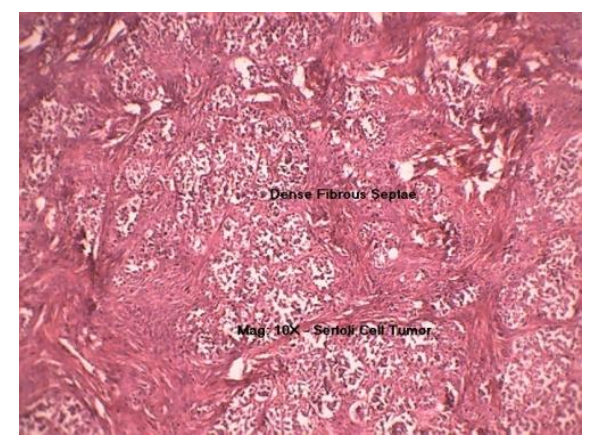

Figure 2: Tubules separated by dense fibrous septae.

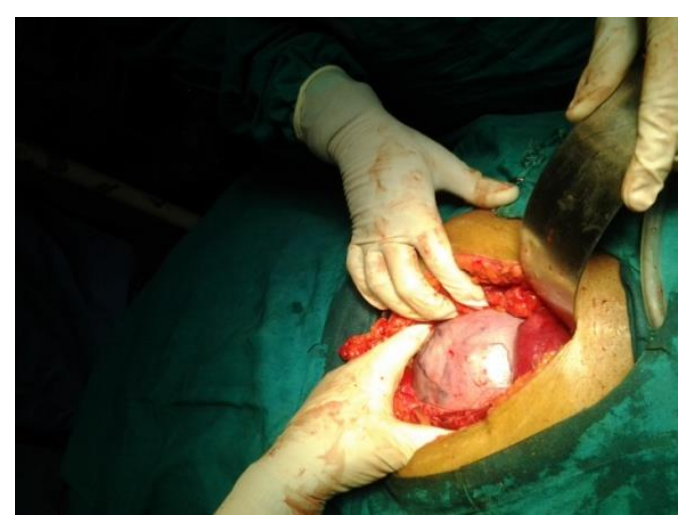

Figure 3: A hard, fixed, vascular mass 20 x $15 \mathrm{~cm}$ arising from right cornua adhered to uterus laterally and posteriorly invading rectovaginal septum and into the retroperitoneal space beneath the leaves of broad ligament almost engulfing the righ. 


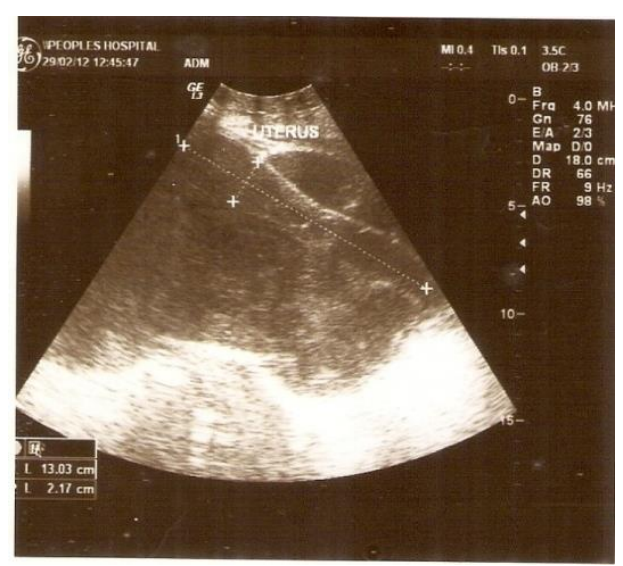

Figure 4: Large malignant right ovarian growth closely adherent to right lateral and posterior wall of the uterus.

\section{DISCUSSION}

Adnexal masses in postmenopausal age group present unique challenges in diagnosis. Due to the age, the concern for potential malignancy is always there. Richard $\mathrm{H}$ et al in their study examined the relationship of menopausal status and stage of the ovarian cancer and found that being postmenopausal conferred a greater likelihood of having any ovarian malignancy $(9.09 \%)$ compared with pre-menopausal women $(4.43 \%))^{3}$ The initial signs and symptoms of ovarian neoplasms at presentation are often non-specific ranging from vague discomfort, pelvic pain, pressure symptoms, anorexia or at times they may be totally asymptomatic to be identified incidentally during a routine clinical examination. ${ }^{4}$ In the present case, the mass remained largely asymptomatic till quite large and was picked up only on clinical examination. The importance of thorough pelvic examination in this age group irrespective of clinical presentation can never be overemphasized.

Besides a thorough pelvic ultrasound, the diagnostic evaluation of such adnexal masses includes an abdomino pelvic computed tomography and wide gamut of tumor markers including hormonal assays. Despite this thorough pre-op screening the risk of encountering an unexpected malignancy ranges from 0.9 to $13 \% .^{5}$ Various scoring systems, biochemical predictors and algorithms have been tried to identify the high risk group and plan the surgery before hand but all have their limitations. High frequency grey scale ultrasonography, most widely used to image such masses lacks the specificity $(0.68$ to $0.81 \%)$ and positive likelihood ratio for cancer prediction (3.3). ${ }^{6}$ Color Doppler though adds color, the values of its indices overlap considerably between benign and malignant masses. ${ }^{6} \mathrm{CT}$ and MRI being costly enough are not considered as primary screening tools and are considered complimentary to grey scale ultrasonography. CA 125, the most extensively studied serum marker in ovarian tumors has a limited role in evaluation of non - epithelial ovarian tumors with a possibility of misleading normal values especially in early stage disease. Normal CA125 values and close adherence of the mass to the posterior wall of the uterus on sonography, in our case kept the provisional diagnosis of degenerating fibroid higher on the list. Also, the fine needle aspiration cytology which was attempted twice suggested nothing but a possible benign spindle cell lesion.

The sex chord stromal tumors are rare and principally seen in $2^{\text {nd }}$ and $3^{\text {rd }}$ decade of life.

The majority of these tumors are functional and characterized by production of variable amount of androgens responsible for virilization or estrogen production responsible for clinical symptoms such as abnormal bleeding patterns. ${ }^{7,8}$ In a study conducted by Bhat RA on sertoli leydig cell tumor of the ovary the median age of the patients was 30 years with $80 \%$ of the patients presenting with hormone related symptoms in the form of secondary amenorrhea, irregular menses and variable degree of virilization. ${ }^{9}$ Vinni Grover reported a case of hilar leydig cell tumor in a 51 year old lady with surgical menopause who had presented with worsening hirsutism. $^{10}$ Occurrence of sertoli cell tumor in postmenopausal age group is a rarity and absence of clinical indicators of hormonal production in our case made it further difficult to be suspected clinically.

Because of the rarity of the ovarian sertoli cell tumors the morphologic spectrum, their behavior and factors influencing the latter are not clearly established. They may be mimicked by many different tumors, some of which may be more frequent than Sertoli cell tumors. Usually they are unilateral, solid and often yellow in color with distinctive tubular pattern that facilitates the diagnosis however other patterns may also predominate, causing difficulty in diagnosis. Olivia $E$ et al in their study of 54 cases of sertoli cell tumor variously recorded other microscopic patterns as trabeculae (28), diffuse (21), pseudopapillary (4), retiform (3), islands or alveolar arrangements (3), and spindled (3). ${ }^{11}$

The role of immunohistochemistry, speculated to aid in diagnosis has not been documented in large series. Sertoli cell tumors behave differently in taking up the immunohistochemical stains as well. In the study by Olivia E. et al 18 of 22 patients showed positivity for Inhibin while 15 of 23 were positive forAE1/3-Cam5.2. ${ }^{11}$ Epithelial membrane antigen (EMA) was negative in all cases in their series. EMA, inhibin, and chromogranin are the three stains commonly employed to exclude other differentials of Sertoli cell tumors, namely endometrioid carcinoma [inhibin-; EMA+; chromogranin-] and Carcinoid tumor [inhibin-; EMA+; chromogranin +]. About $20 \%$ of sex cord tumors show presence of various heterologous elements either endodermal (cysts or glands) or mesenchymal elements (bone, cartilage or skeletal muscle). Heterologous elements and a combination of these tumours with other ovarian tumors have been variously reported in literature. ${ }^{12,13}$ In the background of 
paucity of literature, a high suspicion of such variants while dealing with ovarian tumors would be desirable.

The most important prognostic factors in these tumors are their stage and degree of differentiation. ${ }^{14}$ In a review of 207 cases by Young and Scully, all well-differentiated tumors were benign, whereas $11 \%$ of tumors with intermediate differentiation, $59 \%$ of tumors with poor differentiation, and $19 \%$ of those with heterologous elements were malignant. ${ }^{15}$ The recurrence of malignant sertoli leydig cell, if at all is early and mostly confined to the pelvis and abdomen with distant metastasis very rarely reported. The BEP regimen has been found to be comparatively safe chemotherapeutic regimen in such cases. ${ }^{16}$ Our patient was also offered adjuvant chemotherapy (BEP) on discharge. The factors responsible for this decision were incomplete staging and resection and moderate differentiation on histopathology.

\section{CONCLUSION}

Adnexal masses in postmenopausal women often remain asymptomatic till quite large and careful pelvic examination in this age group is warranted irrespective of the clinical presentation. Sertoli cell tumors known for variable amount of hormone production may have clinical variants and a high suspicion of clinical variants to be kept in mind while dealing such ovarian tumors. In the background of paucity of literature on sertoli cell ovarian tumors, maintenance of central registry of such cases would be highly desirable.

\section{ACKNOWLEDGEMENTS}

The authors express their gratitude to

1) Dr. Rekha Sapkal, Prof. Dept of Obst \& Gyn, P.C.M.S. \& R.C., Bhopal for her expert guidance and support.

2) Dr. Nitin Garg, Asso. Prof., dept of surgery, P.C.M.S. \& R.C., Bhopal for his expert help.

\section{REFERENCES}

1. Jonathan S. B., Sathima Natrajan. Ovarian and Fallopian Tube Cancer. In: Jonathan S. Berek, eds. Berek \& Novak's Gynaecology. 14th ed. Philadelphia, PA: Lippincott Williams \& Wilkins; 2007: 1457-1547.

2. Reed N., Millan D., Verheijen R, et al. Non epithelial ovarian cancer: ESMO Clinical Practice Guidelines for diagnosis, treatment and follow - up. Ann Oncol 2010;21(suppl 5): v31-v36.

3. Demir R.H., Marchand G.J. Adnexal masses suspected to be benign treated with laparoscopy. JSLS. 2012 Jan-Mar; 16(1): 71-84.

4. Rossing MA, Wickland KG, Cushing-Haugen KL, et al. Predictive value of symptoms for early detection of ovarian cancer. J Natl Cancer Inst. 2010;102(4):222-229.

5. Muzi L, Angioli R, Zullo M, Panici PB. The unexpected ovarian malignancy found during operative laparoscopy: incidence, management, and implications for prognosis. J Min Invasive Gynecol. 2005;12:81-89.

6. Lisa Graham. ACOG releases guidelines on management of adnexal masses. Am Fam Phys. 2008 May; 77 (9) :1320-1323.

7. Alam K, Maheshwari V, Rashid S, et al. Bilateral sertoli-leydig cell tumor of the ovary: A rare case report. Indian J Pathol Microbiol 2009;52:97-9.

8. Metwalley KA, Elsers DA, Farghaly HS, et al. Precocious puberty secondary to a mixed germ cell - sex cord stromal tumor associated with an ovarian yolk sac tumor: a case report. J Med Case Rep 2012;6:162.

9. Bhat RA, Lim YK, Chia YN. et al. Sertoli-Leydig cell tumor of the ovary: Analysis of a single institution database. J Obstet Gynaecol Res.2012 June13.doi:10.1111/J 1447-0756.2012.01928.

10. Grover V., Babu A. Hilar leydig cell tumor presenting as hirsutism in a 51 year old woman: Case report. Hospital Physician. Oct 2007;33-38.

11. Olivia E. Alvarej T., Young RH. Sertoli cell tumors of the ovary: a clinicopathological and immunohistochemical study of 54 cases. Am J Surg Pathol.2005 Feb;29(2):143-56.

12. Tandon R., Goel P., Saha P.K. et al. A rare ovarian tumor - sertoli leydig cell tumor with heterologous element. Med Gen Med 2007 Nov; 9(4):44.

13. Jayalakshmy P.S., Usha Poothiode, Krishna G. et al. Ovarian fibroma with serous cystadenoma- An unusual combination: a case report. Case Rep Obstet Gynecol.2012: 2012641085.

14. Fox H, Wells M. Surface epithelial stromal tumors of the ovary. In: Fox H, Wells M, eds. Haines \& Taylor Obstetrical and Gynaecological Pathology. Vol. 1. Madrid, Spain: Churchill Livingstone; 2003.

15. Young RH, Scully RE. Ovarian Sertoli- Leydig cell tumours. A clinicopathological analysis of 207 cases. Am J Surg Pathol. 1985;9:543-569.

16. Gershenson DM, Morris M, Burke TW et al. Treatment of poor-prognosis sex cord-stromal tumors of the ovary with the combination of bleomycin, etoposide, and cisplatin. Obstet Gynecol. 1996;87:527-531.

17. Colombo N, Peiretti M, Castiglione M. Nonepithelial ovarian cancer: ESMO clinical recommendations for diagnosis, treatment and follow - up. Ann Oncol 2009;20(suppl 4)iv24-iv26.

DOI: $10.5455 / 2320-1770$. ijrcog20131246

Cite this article as: Jain M, Modi JN, Chourasia U,

Singhal R. Suspecting the unsuspected in a large adnexal mass in a postmenopausal woman. Int $\mathbf{J}$ Reprod Contracept Obstet Gynecol 2013;2:704-7. 